\title{
Teledermatologia: uma interface entre a atenção primária e atenção especializada em Florianópolis
}

\section{Teledermatology: an interface among primary and specialized care in Florianopolis \\ Teledermatología: una interfaz entre la atención primaria y atención especializada en salud en Florianópolis}

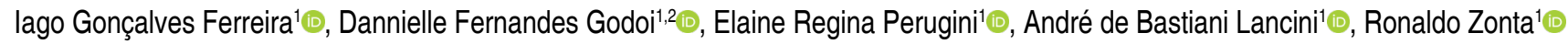 \\ ${ }^{1}$ Escola de Saúde Pública de Florianópolis - SMS Florianópolis. Florianópolis, SC, Brasil. \\ ${ }^{2}$ Universidade Federal de Santa Catarina (UFSC). Florianópolis, SC, Brasil.
}

\section{Resumo}

Introdução: A aplicação do telediagnóstico em dermatologia pode apresentar diversos benefícios como a redução do tempo de espera para consultas com especialistas focais, triagem de doenças graves - principalmente neoplasias - e educação permanente de profissionais. Objetivo: Relatar a experiência da implantação do serviço de teledermatologia no município de Florianópolis - Santa Catarina - e o impacto inicial do processo no acesso e qualificação da assistência aos pacientes com afecções dermatológicas. Métodos: Estudo observacional, transversal e descritivo. Foram utilizados dados do sistema de prontuário eletrônico municipal, base de dados da plataforma Telessaúde-SC e documentos públicos institucionais da Secretaria Municipal de Saúde de Florianópolis entre os anos de 2013 e 2017. Resultados: O serviço de teledermatologia foi implementado no município de Florianópolis em 2015, por meio da adesão ao Sistema Integrado Catarinense de Telemedicina e Telessaúde. O processo de adequação ocorreu por meio da aquisição de equipamentos, adaptações na infraestrutura e capacitações de servidores. No período analisado, verificou-se redução de $52,6 \%$ na taxa de encaminhamentos para dermatologia, com expressiva diminuição no tempo de espera para consulta de cerca de 3 anos, em 2013 e 2014, para 20 dias em 2017. Nota-se tendência à qualificação dos encaminhamentos ao serviço, com redução nos laudos emitidos com classificação azul, manejados na APS, e aumento de laudos com classificação amarela, referenciados para dermatologia. Conclusão: A implantação da teledermatologia em Florianópolis representou um grande avanço para o processo de integração entre a Atenção Primária à Saúde e Atenção Especializada, contribuindo para a redução nas filas de espera e na qualificação dos encaminhamentos, com potencial para o aprimoramento da coordenação do cuidado e educação continuada dos profissionais.

Palavras-chave: Telemedicina; Dermatologia; Atenção Primária à Saúde; Atenção Secundária à Saúde; Medicina de Família e Comunidade

Como citar: Ferreira IG, Godoi DF, Perugini ER, Lancini AB, Zonta R. Teledermatologia: uma interface entre a atenção primária e atenção especializada em Florianópolis. Rev Bras Med Fam Comunidade. 2019;14(41):2003. https://doi.org/10.5712/rbmfc14(41)2003

\author{
Autor correspondente: \\ lago Gonçalves Ferreira. \\ E-mail: iago_goncalves14@ hotmail.com \\ Fonte de financiamento: \\ declaram não haver. \\ Parecer CEP: \\ 2.800.032 (SES/SC), aprovado em \\ 03/08/2018.
}

Procedência e revisão por pares: revisado por pares. Recebido em: 27/02/2019. Aprovado em: 12/04/2019. 


\begin{abstract}
Introduction: The application of tele-diagnosis in dermatology can present several benefits such as waiting time reduction for secondary care, screening for serious diseases - mainly neoplasia - and continuing education of professionals. Objective: Report the experience of tele dermatology service implantation in the municipality of Florianopolis - Santa Catarina, and its initial impact on access and qualification of care for patients with dermatological disorders. Methods: Observational, cross-sectional and descriptive study. The experience report was based on data from municipal electronic medical record system, database from "Telessaude-SC" platform and Municipal Health Department of Florianopolis public institutional documents, among 2013 and 2017. Results: Tele dermatology service was implemented in Florianopolis in 2015, through adherence to Santa Catarina State Integrated Telemedicine (Telemedicina) and Telehealth (Telessaúde) System. The suitability process occurred through equipment acquisition, structural adaptations and staff training. In the analyzed period, there was a reduction of $52.6 \%$ medical referral rate to dermatology, with a significant decrease in waiting time from about 3 years in 2013-2014, for about 20 days in 2017. There is a trend towards the qualification of referrals to the service, with reduction of reports issued with blue classification, handled in Primary Health Care (PHC), and increase of reports with yellow classification, referred to dermatology. Conclusion: Tele dermatology implementation in Florianopolis represented a major advance on Primary Health Care and Specialized Health Care integration process, contributing to waiting times reduction and qualification of referrals, with potential improvement of coordination care and continuing education of professionals.
\end{abstract}

Keywords: Telemedicine; Dermatology; Primary Health Care; Secondary Care; Family Practice

\title{
Resumen
}

Introducción: La aplicación del telediagnóstico en dermatología puede presentar diversos beneficios, como la reducción del tiempo de espera para consultas con expertos en la investigación, para la clasificación de enfermedades graves - principalmente neoplasias - y educación permanente de profesionales. Objetivo: Relatar la experiencia de la implantación del servicio de teledermatología en el municipio de Florianópolis - Santa Catarina y su impacto inicial del proceso en el acceso y cualificación de la asistencia a los pacientes con afecciones dermatológicas. Métodos: Estudio observacional, transversal y descriptivo. Para relatar la experiencia se utilizó datos del registro medico electrónico municipal, base de datos de la plataforma "Telessaúde-SC" y documentos públicos institucionales de la Secretaría Municipal de Salud de Florianópolis entre los años 2013 y 2017. Resultados: El servicio de teledermatología fue implementado en el municipio de Florianópolis en 2015, a través de la adhesión al Sistema Integrado Catarinense de Telemedicina y Telessaúde. El proceso de adecuación ocurrió por medio de la adquisición de equipamientos, adaptaciones en la infraestructura y capacitaciones de servidores. En el período analizado, se verificó reducción de 52,6\% en la tasa de encaminamientos para dermatología con expresiva disminución en el tiempo de espera para consulta de cerca de 3 años en 2013 y 2014 para 20 días en 2017 . Se observa tendencia a la cualificación de los encaminamientos al servicio, con reducción de los laudos emitidos con clasificación azul, manejados en la APS, y aumento de laudos con clasificación amarilla, encaminados a dermatología. Conclusión: La implantación de la teledermatología en Florianópolis representó un gran avance para el proceso de integración de la Atención Primaria a la Salud y la Atención Especializada, contribuyendo con la reducción de las colas de espera y en la cualificación de los encaminamientos, con potencial para el mejoramiento de la coordinación del cuidado y la educación continuada de los profesionales.

Palabras clave: Telemedicina; Dermatología; Atención Primaria de Salud; Atención Secundaria de Salud; Medicina Familiar y Comunitaria

\section{Introdução}

As tecnologias de informação e comunicação têm revolucionado as interações sociais e compartilhamento de conteúdo nas últimas décadas, apresentando grande potencial para o enfrentamento dos problemas de saúde globais. Tais tecnologias podem contribuir para a melhoria do acesso a recursos de melhor qualidade na saúde, desenvolvimento de pesquisas e educação em saúde, beneficiando principalmente países em desenvolvimento. ${ }^{1}$

Nessa perspectiva, a telemedicina apresenta-se como um recurso tecnológico que permite a transferência e utilização de informações em saúde com finalidades diagnóstica, terapêutica e de educação permanente de profissionais, possibilitando a interação entre diferentes serviços e níveis de atenção à saúde. ${ }^{2-4}$ A telemedicina apresenta diversas aplicações nos serviços de saúde como: teleconsultorias, telecirurgia, teleducação, videoconferências e o telediagnóstico, adequando-se às necessidades e aos recursos disponíveis. ${ }^{5}$ 
Apesar das distintas possibilidades de aplicação, a telemedicina ainda desperta questões controversas acerca de seu uso. Tais demandas relacionam-se à carência de políticas que regulem a privacidade e a confidencialidade dos pacientes, a atribuição de responsabilidade médica acerca do compartilhamento e transferências de dados clínicos, além da falta do exame físico e contato direto com os pacientes. ${ }^{6,7}$

Objetivando regulamentar o uso da telemedicina no Brasil, o Conselho Federal de Medicina publicou a resolução no 2.227/2018, que estabelece requisitos e normas para o tráfego de dados e imagens de pacientes por meio do uso de tecnologias de informação. ${ }^{8}$ Entretanto, após o recebimento de alto número de manifestações de médicos brasileiros e entidades representativas da classe, o Conselho decide revogar a resolução para recebimento de contribuições, reavaliação do tema e reformulação do documento. ${ }^{9}$

A despeito do debate e controvérsias, a telemedicina revela-se como uma nova possibilidade de integração entre médicos da atenção primária e atenção especializada, permitindo o intercâmbio de informações e qualificação profissional, por meio da solução de dúvidas diagnósticas e apoio às condutas terapêuticas. ${ }^{3,10,11}$ Ademais, ainda demonstra o potencial de reduzir a demanda por consultas presenciais e a necessidade de deslocamentos de pacientes ao permitir, por exemplo, a realização de exames complementares nas próprias unidades básicas de saúde ou em serviços de suporte, com laudos realizados por especialistas correlatos, como no telediagnóstico. . $^{10,12,13}$

À vista disso, a dermatologia demonstra-se uma área propícia para a aplicação dos recursos de telediagnóstico. Por meio da captura e transmissão de fotografias digitais (macroscopia) e/ou dermatoscopia digital (com ou sem luz polarizada), a teledermatologia permite a execução de exames à distância, sob a forma de três modelos de sistemas: store-and-forward, ou sistema assíncrono, modelo em que as imagens são obtidas seguindo protocolos pré-estabelecidos, sendo armazenadas e enviadas, juntamente com informações clínicas dos pacientes como tempo de evolução do quadro, sintomas e tamanho das lesões, para posterior avaliação por dermatologistas. Sistema real-time, ou síncrono, em que a avaliação dermatológica ocorre em tempo real através de videoconferência entre paciente, dermatologista e médico generalista, permitindo comunicação simultânea. Modelo híbrido, representado pela mescla dos dois sistemas. $^{12-16}$

Além da possibilidade de diagnóstico das lesões, a plataforma da teledermatologia apresenta também benefício educacional, ${ }^{16}$ na medida em que o feedback entre profissionais, mais rápido que processos tradicionais de consulta, permite a classificação de risco e orientação da conduta clínica ou eventual necessidade de avaliação presencial especializada. Desta forma, todo o processo atua como ação diagnóstica, de regulação do acesso à atenção especializada e, adicionalmente, de educação permanente para os profissionais envolvidos.

A primeira experiência no uso da teledermatologia ocorreu pelo Departamento de Defesa dos Estados Unidos com soldados na missão "Restore Hope" na Somália, em 1992. Durante o período de um ano, 74 imagens foram transmitidas com auxílio de transceptor satélite portátil, oferecendo assistência dermatológica aos combatentes. ${ }^{15}$ No Brasil, a experiência pioneira ocorreu por meio do Projeto Telederma desenvolvido pelo Serviço de Dermatologia do Hospital das Clínicas de Porto Alegre e a disciplina de Telemedicina da Faculdade de Medicina da Universidade de São Paulo em 2002, com envolvimento de graduandos, residentes e dermatologistas. ${ }^{4}$ 
Diante da relevância das possibilidades e recursos da telemedicina para a assistência à saúde no âmbito da Atenção Primária à Saúde, assim como, das experiências exitosas de implementação da modalidade teledermatologia, o município de Florianópolis iniciou o processo de implantação desse serviço no ano de 2015.

\section{Panorama da rede municipal de saúde de Florianópolis}

O município de Florianópolis, capital do estado de Santa Catarina, tem população estimada em 492.977 habitantes, com índice de desenvolvimento humano municipal (IDHM) de 0,847. ${ }^{17}$ A rede municipal de saúde apresenta a Atenção Primária à Saúde composta por 49 centros de saúde, como modelos de atenção exclusivamente formado pela estratégia de saúde da família (ESF), com cobertura de ESF variando de 88 a $100 \%$ no período avaliado por este estudo. ${ }^{18}$ Os centros de saúde estão distribuídos em quatro distritos: centro, norte, continente e sul. Na Atenção Especializada, a rede possui quatro policlínicas municipais, uma por distrito de saúde, com oferta de diferentes especialidades médicas e de outras áreas profissionais.

\section{O acesso à Atenção Especializada: um desafio para Florianópolis}

O acesso à Atenção Especializada/média complexidade apresenta-se como um importante desafio para a garantia da integralidade da atenção no SUS. Este desafio engloba a transposição de diversos obstáculos como a falta de integração e articulação entre os níveis de atenção, a precariedade na oferta e qualidade dos serviços, o subfinanciamento e a fragilidade na gestão das filas de espera. ${ }^{11} \mathrm{O}$ aporte financeiro para a saúde, apesar de importante pilar para a melhoria da qualidade do acesso, não representa a "solução" para esses entraves, tendo em vista que os recursos são finitos e que a racionalidade e eficiência na aplicação destes faz-se imperativa.

Florianópolis também faz parte desse cenário desafiador para a saúde pública brasileira, o qual pode ser ilustrado pela condição da fila de espera para a especialidade de dermatologia, um dos casos mais graves registrados no Sistema de Regulação (SISREG) do município em 2015, que apresentava cerca de 4000 usuários na fila de espera por consulta. Esse quantitativo, considerando a oferta disponível no serviço de dermatologia do município, representava um tempo de espera de quase três anos para o atendimento na especialidade.

Nessa conjuntura, um dos principais problemas, além do longo tempo de espera para as consultas, consistia na carência de critérios clínicos que justificassem muitos dos encaminhamentos para a dermatologia. Segundo levantamentos realizados pela equipe técnica de especialistas à época, cerca de $50 \%$ dos casos não apresentava indicação para consulta especializada. Por essa compreensão, Gomes et al. ${ }^{11}$ ressaltam a importância que os médicos da APS sejam capazes de realizar o diagnóstico e o acompanhamento das afecções da pele mais comuns, como acne, infecções fúngicas e bacterianas, assim como, reconhecer lesões de pele com sinais indicativos de malignidade e afecções mais complexas que necessitam de avaliação especializada focal.

Sendo assim, torna-se necessária a ampliação da comunicação entre serviços e especialidades, promovendo uma abordagem integral, de maneira a qualificar os profissionais e aprimorar o grau de resolutividade da APS. ${ }^{11}$ Frente a esta conjuntura, as equipes de gestão da Média Complexidade e Atenção Primária da Secretaria Municipal de Florianópolis articularam-se em um processo de gestão de filas de 
espera, tendo a dermatologia como primeira especialidade para implementação dessa iniciativa. Dentre as possibilidades avaliadas, o serviço de teledermatologia da Secretaria de Estado de Saúde de Santa Catarina revelou-se uma alternativa viável e estratégica.

Dessa maneira, este estudo teve como objetivo relatar o processo de implementação dessa tecnologia, bem como, os impactos ocasionados nas filas de agendamentos de consultas, capacitação profissional e qualidade do atendimento, analisando o quinquênio 2013 a 2017.

\section{Métodos}

O estudo foi conduzido baseando-se nos preceitos da Declaração de Helsinque e do Código de Nuremberg, bem como em consonância com as Normas de Pesquisas Envolvendo Seres Humanos da Comissão Nacional de Ética em Pesquisa (Resolução CONEP 466/2012). O levantamento de dados teve início após a aprovação do Comitê de Ética em Pesquisa com Seres Humanos da Secretaria de Estado de Saúde de Santa Catarina (CAAE: 91700318.3.0000.0115), bem como, aprovação da Comissão de Acompanhamento de Projetos de Pesquisa em Saúde da Secretaria Municipal de Saúde de Florianópolis e Gerência do Núcleo Estadual de Telemedicina de Santa Catarina, instituições na quais os dados do estudo foram levantados.

Trata-se de um relato de experiência, por meio de levantamento de dados em perspectiva descritiva, observacional, quantitativa e retrospectiva. A descrição da experiência foi elaborada a partir da coleta de dados secundários referentes aos anos de 2013 a 2017. Os dados foram extraídos a partir dos sistemas: Infosaúde, da Secretaria Municipal de Saúde de Florianópolis, composto por registros de atendimentos na Atenção Primária à Saúde (APS) e Atenção Especializada; Sistema Catarinense de Telemedicina e Telessaúde, do Núcleo Estadual de Telemedicina - Secretaria de Estado de Saúde de Santa Catarina, que armazena os registros de laudos de teledermatologia; e Sistema de Centrais de Regulação (SISREG), que contém informações acerca das solicitações inseridas e agendadas para a especialidade no município de Florianópolis. Além desses bancos de dados, foram incluídos na análise relatórios e documentos oficiais da Gerência de Atenção Especializada da Secretaria Municipal de Saúde de Florianópolis.

Os dados foram analisados com método descritivo, tendo as variáveis categóricas e quantitativas representadas por meio de gráficos e tabelas desenvolvidos nos programas Microsoft Excel ${ }^{\circledR} 2016$ e Microsoft Word ${ }^{\circledR} 2016$.

\section{Resultados}

\section{O serviço de telemedicina em Santa Catarina}

O processo de implementação do serviço de telemedicina no estado de Santa Catarina teve início no ano de 2005 por meio de uma iniciativa entre a Universidade Federal de Santa Catarina (UFSC) e a Secretaria de Estado de Santa Catarina (SES-SC), resultando na construção de uma plataforma de software de comunicação e transmissão de imagens e dados. ${ }^{12,19} \mathrm{O}$ sistema adotou o modelo store-and-forward, ou sistema assíncrono, em que as imagens são capturadas com auxílio de dermatoscópios e câmeras digitais, sendo armazenadas e enviadas para análise e laudo diagnóstico de dermatologistas. ${ }^{19}$ 
Os laudos são emitidos de acordo com critérios de estratificação de risco elaborados pelo Núcleo Estadual de Telemedicina/SC, de forma a orientar os fluxos do sistema e as condutas em relação aos quadros clínicos. A classificação é organizada em cinco cores: branca, azul, verde, amarela e vermelha, evoluindo em critérios de gravidade, sendo a branca caracterizada como sem necessidade de intervenção e encaminhamento, até a vermelha, representando os casos com critérios de gravidade e necessidade de referenciamento imediato a um serviço de emergência. ${ }^{19}$

O município de Florianópolis decidiu vincular-se ao Sistema de Telemedicina para a especialidade de dermatologia em abril de 2015, iniciando assim um processo de capacitação de servidores e implementação do serviço na rede.

\section{A implementação da teledermatologia: articulando os níveis de atenção}

O planejamento das intervenções necessárias para implementação da plataforma de teledermatologia ocorreu mediante a articulação de um grupo de trabalho formado por profissionais da gestão, Atenção Primária à Saúde e Média Complexidade, dermatologistas e médicos de família e comunidade, em parceria com o Núcleo Estadual de Telemedicina/SC. O fluxo de funcionamento do processo seguiu o protocolo estabelecido pelo Sistema Catarinense de Telemedicina vinculado à Secretaria Estadual de Saúde (Figura 1).

O processo foi executado em várias etapas, incluindo: implementação do exame de dermatoscopia nas quatro policlínicas municipais (treinamento da equipe de enfermagem no uso do equipamento e captação das imagens e treinamento dos dermatologistas da rede na elaboração dos laudos); cadastro e capacitação de todos os médicos das equipes de saúde da família (ESF) na plataforma do Sistema Integrado Catarinense de Telemedicina e Telessaúde; revisão dos encaminhamentos pendentes em fila de espera para dermatologia; e estabelecimento de equipe de apoio e monitoramento da implantação teledermatologia.

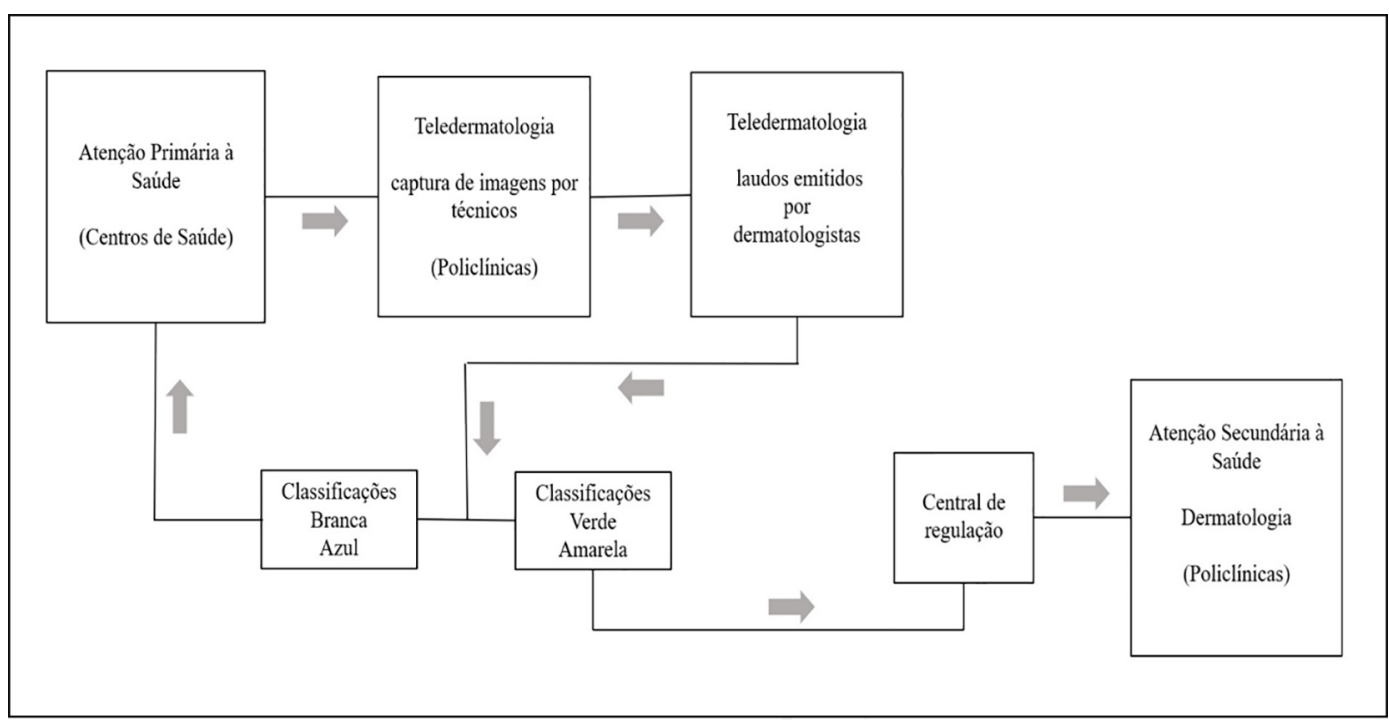

Figura 1. Fluxograma do processo regulatório entre Atenção Primária, Teledermatologia e Dermatologia. Fonte: elaborado pelos autores. 
Vale ressaltar que, paralelamente ao processo de implantação da teledermatologia no ano de 2015, uma equipe técnica formada por médicos de família e dermatologistas realizou a análise de todos encaminhamentos pendentes em fila de espera. Os encaminhamentos pendentes em fila espera foram classificados de acordo com os dados clínicos e critérios de prioridade, sendo agendados na especialidade, ou devolvidos para reavaliação nas Unidades de Saúde e posterior inserção no sistema se indicado. A devolução dos encaminhamentos pendentes para reavaliação pelas equipes da APS representou uma etapa fundamental do processo, possibilitando a oferta de consultas para pacientes já avaliados pela teledermatologia, resultando em diminuição drástica no tempo de espera para a especialidade.

\section{Impacto no acesso à dermatologia na rede municipal de saúde de Florianópolis}

Após a análise dos dados extraídos dos relatórios do sistema de prontuário eletrônico (Infosaúde) referentes ao período de 2013 a 2017, identificou-se redução na taxa de encaminhamentos gerados para especialidade após a implantação do serviço de teledermatologia em 2015. No ano de 2013, registrou-se taxa de encaminhamentos para dermatologia de 6,17 a cada 1000 consultas médicas realizadas na APS e 2,92 encaminhamentos para cada 1000 consultas médicas em 2017, dados esses que representam uma redução de $52,6 \%$ no período (Tabela 1 ).

Tabela 1. Encaminhamentos da Atenção Primária à Saúde para a Atenção Especializada em dermatologia no município de Florianópolis, quinquênio 2013 - 2017.

\begin{tabular}{|c|c|c|c|c|c|c|c|c|c|}
\hline \multirow[t]{2}{*}{ Anos } & \multirow{2}{*}{$\begin{array}{c}\text { Atendimentos } \\
\text { APS }\end{array}$} & \multicolumn{2}{|c|}{ Dermatologia } & \multicolumn{2}{|c|}{$\begin{array}{c}\text { Dermatologia } \\
\text { (Pediatria) }\end{array}$} & \multicolumn{2}{|c|}{$\begin{array}{l}\text { Dermatologia } \\
\text { (Hanseníase) }\end{array}$} & \multicolumn{2}{|c|}{ Teledermatologia } \\
\hline & & (n) & Taxa* & (n) & Taxa* & (n) & Taxa* & (n) & Taxa* \\
\hline 2013 & 525528 & 3247 & 6,17 & 589 & 1,12 & 26 & 0,04 & 0 & 0 \\
\hline 2014 & 576805 & 3681 & 6,38 & 736 & 1,27 & 28 & 0,04 & 0 & 0 \\
\hline 2015 & 580643 & 1894 & 3,26 & 555 & 0,95 & 27 & 0,04 & 1872 & 3,22 \\
\hline 2016 & 615378 & 1684 & 2,73 & 412 & 0,66 & 27 & 0,04 & 3718 & 6,04 \\
\hline 2017 & 564149 & 1651 & 2,92 & 724 & 1,28 & 26 & 0,04 & 3435 & 6,08 \\
\hline Total & 2862503 & 12157 & & 3016 & & 134 & & 9025 & \\
\hline
\end{tabular}

(n): quantitativo de encaminhamentos; * número de encaminhamentos por 1000 atendimentos médicos na APS. Fonte: Banco de dados Infosaúde - Secretaria Municipal de Saúde de Florianópolis.

Os dados obtidos por meio do banco de dados Infosaúde apresentaram tendências coincidentes com os dados do Sistema de Centrais de Regulação (SISREG), acerca do quantitativo de encaminhamentos e inserções no sistema para atendimentos em dermatologia e teledermatologia. Ao se observar os dados do SISREG, nota-se redução de cerca de $50 \%$ no quantitativo de inserções para dermatologia no sistema, no comparativo entre o primeiro semestre de 2013 e o segundo semestre de 2017 (Figura 2).

Em relação à lista de espera para a especialidade de dermatologia, nos anos de 2013 e 2014, o quantitativo de pacientes aguardando era 4080 e 4085, respectivamente, com tempo de médio de espera de mais de 1000 dias. Após a implementação do serviço de teledermatologia em 2015, associado ao processo de reavaliação dos encaminhamentos pendentes em fila, observou-se queda expressiva no número de pacientes nas listas e no tempo de espera, atingindo 98 pacientes na fila e 20 dias de espera em 2017, identificando-se tendência ao equilíbrio entre demanda e oferta de atendimentos em dermatologia (Figura 3). 


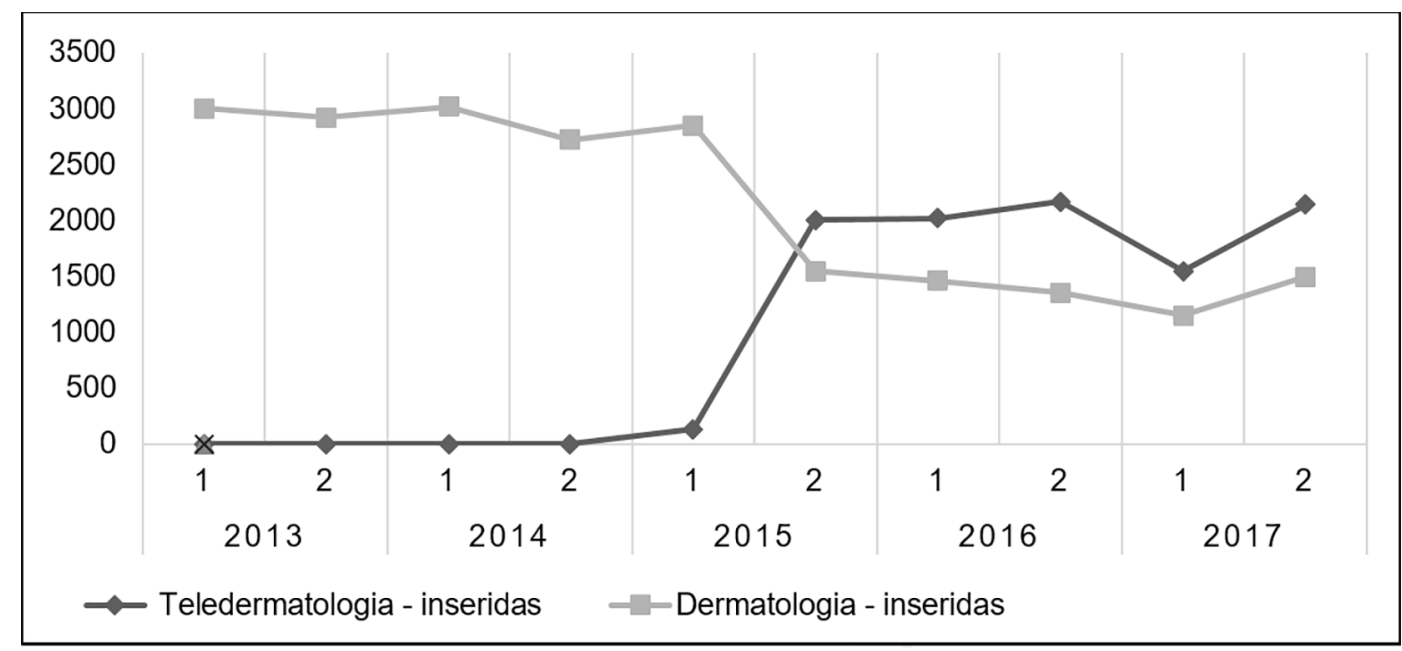

Figura 2. Solicitações para dermatologia e teledermatologia inseridas no Sistema de Centrais de Regulação - SISREG, por semestres, no quinquênio 2013 - 2017.

Fonte: Banco de dados SISREG - Secretaria Municipal de Saúde de Florianópolis.

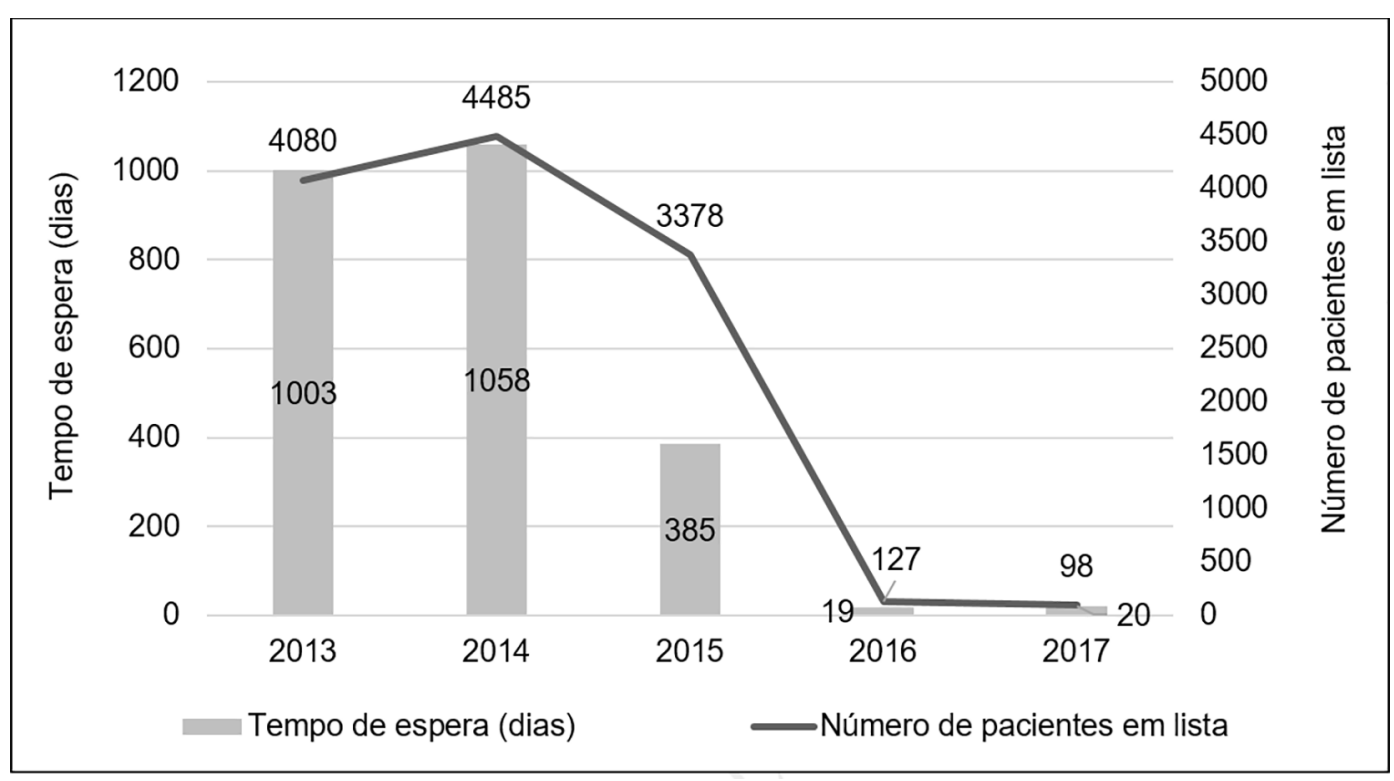

Figura 3. Evolução do quantitativo de pacientes aguardando em lista de espera para consulta em Dermatologia e tempo e espera no município de Florianópolis, quinquênio 2013 - 2017. Fonte: Banco de dados SISREG - Secretaria Municipal de Saúde de Florianópolis.

Quanto aos encaminhamentos ao serviço, nota-se aumento no total de atendimentos no serviço ao se comparar os anos de 2015 e 2016, em todas as classificações de risco, com destaque para as classificações branca, com aumento de 479 para 1032, e verde, de 405 para 1181. Entretanto, ao analisar-se os anos de 2016 e 2017, identifica-se redução nos valores absolutos em todas as classificações, com exceção da amarela, referenciados para dermatologia (Figura 4).

Quanto aos valores relativos entre os anos de 2015 e 2017, verifica-se tendência à redução na porcentagem de laudos com classificação azul, de 14,85\% em 2015 para 9,1\% em 2017; e aumento gradual nos laudos com classificação amarela, de 21,41\% em 2015 para 30,56\% em 2017 (Figura 4). 


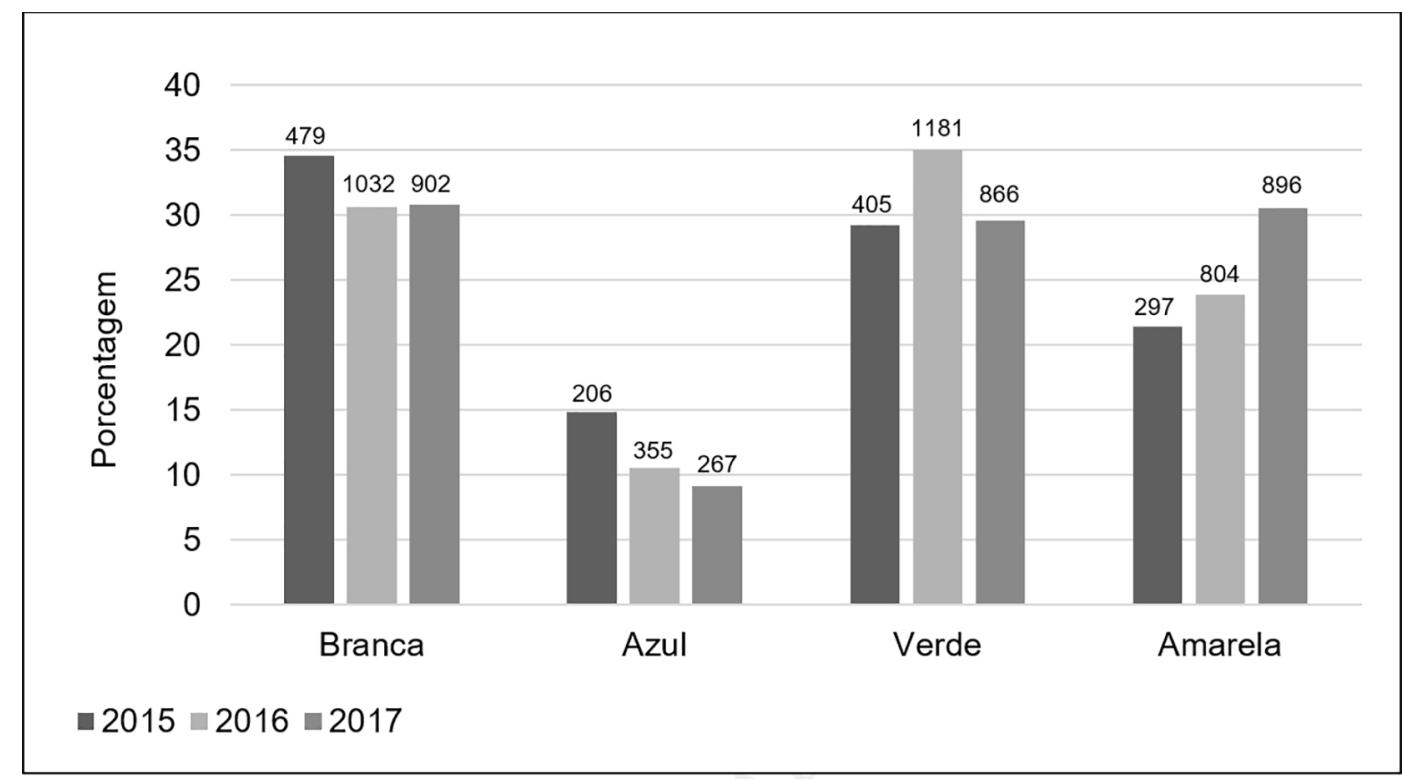

Figura 4. Classificações* dos laudos do serviço de teledermatologia em Florianópolis, em porcentagens, no período de 2015 a 2017.

Fonte: Banco de dados Telemedicina - Secretaria de Estado de Saúde de Santa Catarina.

* A classificação vermelha não foi representada no gráfico, pois houve apenas um laudo classificado com prioridade vermelha no período, em 2016.

\section{Discussão}

O acesso à atenção especializada, ou à média complexidade, representa um dos principais desafios para a consolidação da integralidade da atenção no âmbito do SUS. Por esse aspecto, os sistemas de regulação desempenham papel estratégico e fundamental na organização dos fluxos entre APS e Atenção Especializada por meio de protocolos preestabelecidos, gerenciam solicitações de exames, agendamentos, laudos e priorizando a oferta em tempo oportuno de acordo com critérios de gravidade padronizados. ${ }^{12,20}$

Por meio dos resultados levantados por este estudo, pôde-se observar que a implantação do serviço de teledermatologia propiciou redução na taxa de encaminhamentos para especialidade de dermatologia, com consequente diminuição no quantitativo de pacientes em listas de espera para consulta, bem como, no tempo de espera, corroborando com Whited, ${ }^{16}$ que verificou taxa média de $50 \%$ de consultas evitadas na modalidade store-and-forward.

Nessa perspectiva, infere-se que a aplicação da modalidade de telediagnóstico em dermatologia pode apresentar potencialidades como a redução do tempo de espera para consultas com especialistas focais, triagem e priorização de doenças graves (especialmente neoplasias), possibilitando o acesso em tempo oportuno à especialidade. ${ }^{6,14,21}$

Nesse estudo, observa-se ainda tendência à qualificação no perfil de encaminhamentos dos médicos da APS ao serviço de teledermatologia, com redução no número relativo de laudos com classificação azul - manejados na APS, bem como, no número absoluto das classificações branca, azul e verde, de menor complexidade. Por outro lado, nota-se aumento, tanto relativo quanto absoluto, em laudos com a classificação amarela - referenciados à dermatologia com prioridade. 
Por esse panorama, estudos ${ }^{16,22}$ apontam o benefício da teledermatologia no manejo de casos de baixa complexidade, que podem ser assistidos na Atenção Primária com base nas recomendações de dermatologistas. Tandjung et al. ${ }^{23}$ demonstraram $36,8 \%$ de laudos com indicativo de encaminhamento à dermatologia em levantamento realizado no Institute of Primary Care, em Zurique - Suíça, o que converge com o índice observado em Florianópolis, onde o perfil de classificação encontrado ao longo do período avaliado demonstra a convergência para encaminhamentos qualificados e com real necessidade de avaliação pelo especialista focal.

Ainda sob esse prisma, Coates et al. ${ }^{6}$ afirmam que o uso do telediagnóstico tende a diminuir na medida em que os médicos na APS ampliam seu conhecimento, configurando-se como um instrumento de capacitação dos recursos humanos da rede de saúde. A mudança no perfil de classificação obtida pela teledermatologia no período estudado, além da redução expressiva da taxa de encaminhamentos para a dermatologia, mostra a efetividade da ferramenta no processo de educação permanente dos profissionais.

Apesar dos resultados inequívocos mostrados por este estudo, respaldados pela literatura internacional, há que se ressaltar que a responsabilidade em gerar e enviar requisições por uma plataforma adicional (Telessaúde) ao sistema de prontuários eletrônico pode representar um desafio à implementação da teledermatologia, tendo em vista o volume de atendimentos e atribuições das equipes de saúde da família e a alteração nos fluxos de trabalho que a uma "nova" função pode ocasionar. Por conseguinte, o suporte técnico completo e contínuo aos profissionais e infraestrutura organizacional apropriada, com apoio de técnicos treinados para captura das imagens e para alimentação de dados no sistema, pode contribuir para atenuar as demandas das equipes e tornar o serviço mais ágil e eficaz. ${ }^{7,16}$

De acordo com Tensen et al., ${ }^{7}$ a percepção das limitações e dificuldades para implementação do serviço pode ser compreendida de maneira distinta por médicos da atenção primária e dermatologistas. Custos, gerenciamento e treinamento da equipe podem representar barreiras à implementação, na percepção de médicos da atenção primária, enquanto responsabilidade legal, confiabilidade do diagnóstico e acompanhamento de casos podem representar obstáculos para dermatologistas.

Outros aspectos a serem considerados referem-se à qualidade das imagens capturadas e adequada história clínica descrita nos encaminhamentos, requisitos fundamentais a serem atendidos pelos serviços de teledermatologia. ${ }^{6}$ Entretanto, a satisfação de tais requisitos representa um grande desafio para a consolidação dessa ferramenta, o que poderá ser equacionado por meio da evolução das tecnologias de captura de imagens e aprimoramento do registro e transmissão de dados. Cabe ressaltar a importância da avaliação médica presencial para efetividade do serviço de teledermatologia, visto que a realização de anamnese e exame físico adequados são fundamentais para o correto encaminhamento de casos e avaliação dos dermatologistas.

Sob esse ângulo, Yarak et al., ${ }^{24}$ por meio de revisão sistemática, apontam a consulta presencial com confirmação histopatológica como padrão ouro para o diagnóstico dermatológico. Apesar disso, ressaltam que estudos dermatológicos comparando a acurácia e/ou concordância do diagnóstico primário clínico e/ou com os diagnósticos clínicos diferenciais com a consulta presencial - mesmo sem confirmação histopatológica - apresentaram elevada concordância, em torno de $60 \%$ a $90 \%$. 
Durante muito tempo, o emprego da teledermatologia esteve limitado aos grandes centros médicos acadêmicos ou a provedores de saúde integrados, contribuindo para a redução de custos, a organizando de fluxos e triagem de pacientes. Entretanto, os avanços na tecnologia de smartphone e a expansão da internet banda larga permitiu a transmissão de vídeos e imagens com baixo custo e alta qualidade, permitindo a utilização de smartphones na avaliação de lesões cutâneas (com ou sem dermatoscópio), incluindo o rastreamento do câncer de pele. ${ }^{25}$ Nesse sentido, o uso desses equipamentos tem sido avaliado e discutido pela Gerência de Atenção Especializada e Secretaria Municipal de Saúde como uma maneira de facilitar o compartilhamento e captura de imagens, otimizando o serviço.

Vale destacar que, paralelamente ao aumento do uso de smartphones, expande-se também o mercado de aplicativos móveis, que se propõem a realizar o diagnóstico e a triagem de lesões de pele utilizando algoritmos automatizados. Estes recursos podem representar um risco para indivíduos leigos, na medida em que estes possuem fácil acesso, encontrando-se disponíveis para download gratuito ou a baixo custo, mas que não estão sujeitos à supervisão técnica da prática teledermatológica clínica ou tradicional. ${ }^{15}$ Dessa forma, evidencia-se a importância do monitoramento e da avaliação da qualidade dos serviços de teledermatologia, a fim de possibilitar a potencialização da aplicação dessa tecnologia de modo eficaz e seguro.

\section{Considerações finais}

A teledermatologia pode representar uma resposta às longas listas de espera por consultas com especialidades focais, representando um modelo de triagem em ambientes ambulatoriais e de internação. ${ }^{6} \mathrm{~A}$ adequada performance de sistemas de telediagnóstico resulta em menor necessidade de encaminhamentos para consultas presenciais, otimizando o acesso a tratamentos específicos, o que minimiza os custos sociais decorrentes do retardo nos diagnósticos e condutas médicas. ${ }^{14}$

A implantação da teledermatologia na rede municipal de saúde de Florianópolis representou um grande avanço para o processo de integração entre a Atenção Primária e Atenção Especializada. As consequências desse recurso inovador podem despontar em curto e médio prazos, por meio da redução nas filas de espera, ampliação do acesso dos usuários à dermatologia, e organização de fluxos, como elucidados no relato. A longo prazo, a teledermatologia tem o potencial de propiciar o aprimoramento da coordenação do cuidado dos usuários e o fortalecimento do arranjo de rede de saúde, contribuindo para a qualificação e atualização dos profissionais mediante a construção coletiva de conhecimento.

Este estudo demonstra resultados expressivos na ampliação do acesso em tempo oportuno à dermatologia, qualificação da assistência às condições dermatológicas mais prevalentes na APS, assim como o potencial uso da teledermatologia como ferramenta de educação permanente.

\section{Contribuição dos autores}

Concepção e/ou delineamento do estudo: IGF, DFG. Aquisição e interpretação de dados: IGF, DFG, ERP, ABL. Análise de resultados: IGF, DFG, ERP, RZ. Redação preliminar: IGF, DFG, ERP. Revisão crítica da versão preliminar: IGF, DFG, ERP, RZ.

Todos os autores aprovaram a versão final e concordaram com prestar contas sobre todos os aspectos do trabalho. 


\section{Agradecimentos}

Os autores agradecem aos servidores da Gerência de Inteligência e Informação da Secretaria Municipal de Saúde de Florianópolis e aos servidores do Núcleo de Telemedicina da Secretaria de Estado de Santa Catarina, pela colaboração e disponibilidade no processo de extração dos bancos de dados. Agradecem também aos servidores da Gerência de Atenção Especializada da Secretaria Municipal de Saúde de Florianópolis, pela disponibilidade e acesso às informações necessárias para elaboração do estudo.

\section{Conflitos de interesses}

Declaram não haver.

\section{Referências}

1. World Health Organization. Telemedicine: opportunities and developments in member states. Report on the second global survey on eHealth. Geneva: World Health Organization; 2010.

2. Almino MAFB, Rodrigues SR, Barros KSB, Fonteles AS, Alencar LBL, Lima LLD, et al. Telemedicina: um instrumento de educação e promoção da saúde pediátrica. Rev Bras Educ Med. 2014;38(3):397-402. https://doi.org/10.1590/S0100-55022014000300015

3. Piropo TGN, Amaral HOS. Telessaúde, contextos e implicações no cenário baiano. Saúde Debate. 2015;39(104):279-87. https://doi. org/10.1590/0103-110420151040413

4. Soirefmann M, Blom MB, Leopoldo LR, Cestari TF. Telemedicina: uma revisão da literatura. Rev HCPA. 2008;28(2):116-9.

5. Rezende EJC, Melo MCB, Tavares EC, Santos AF, Souza C. Ética e telessaúde: reflexões para uma prática segura. Rev Panam Salud Publica. 2010;28(1):58-65. https://doi.org/10.1590/S1020-49892010000700009

6. Coates SJ, Kvedar J, Granstein RD. Teledermatology: from historical perspective to emerging techniques of the modern era: part I: History, rationale, and current practice. J Am Acad Dermatol. 2015;72(4):563-74. https://doi.org/10.1016/j.jaad.2014.07.061

7. Tensen E, van der Heijden JP, Jaspers MW, Witkamp L. Two Decades of Teledermatology: Current Status and Integration in National Healthcare Systems. Curr Dermatol Rep. 2016;5:96-104. https://doi.org/10.1007/s13671-016-0136-7

8. Brasil. Conselho Federal de Medicina [Internet]. Resolução no 2.227 de 13 de dezembro de 2018. Dispõe sobre a telemedicina como forma de prestação de serviços médicos mediados por tecnologias [Acesso 2019 Fev 5]. Disponível em: http://www.portal.cfm.org.br/ images/PDF/resolucao222718.pdf

9. Brasil. Conselho Federal de Medicina [Internet]. Nota informativa. Conselheiros do CFM revogam a Resolução no 2.227/2018, que trata da Telemedicina. [Acesso 2019 Fev 24]. Disponível em: https://portal.cfm.org.br/index.php?option=com_content\&view=article\& id=28096:2019-02-22-15-13-20\&catid=3

10. Nunes AA, Bava MCGC, Cardoso CL, Mello LM, Trawitzki LVV, Watanabe MGC, et al. Telemedicina na Estratégia de Saúde da Família: avaliando sua aplicabilidade no contexto do PET Saúde. Cad Saúde Coletiva. 2016;24(1):99-104. https://doi.org/10.1590/1414$462 \times 201600010187$

11. Gomes TM, Moura ATMS, Aguiar AC. Dermatologia na atenção primária: um desafio para a formação e prática médica. Rev Bras Educ Med. 2012;36(1):125-8. https://doi.org/10.1590/S0100-55022012000100017

12. Brasil. Ministério da Saúde. Manual de Telessaúde para Atenção Básica / Atenção Primária à Saúde. Brasília: Ministério da Saúde, 2012.

13. Mariani AW, Pêgo-Fernandes PM. Telemedicine: a technological revolution. Sao Paulo Med J. 2012;130(5):277-8. https://doi.org/10.1590/ S1516-31802012000500001

14. Miot AH, Paixão MP, Wen CL. Teledermatologia: Passado, presente e futuro. An Bras Dermatol. 2005;80(5):523-32. https://doi.org/10.1590/ S0365-05962005000600011

15. Lee JJ, English JC 3rd. Teledermatology: A Review and Update. Am J Clin Dermatol. 2018;19(2):253-60. https://doi.org/10.1007/s40257017-0317-6 
16. Whited JD. Teledermatology. Med Clin North Am. 2015;99(6):1365-79. https://doi.org/10.1016/j.mcna.2015.07.005

17. Brasil. Instituto Brasileiro de Geografia e Estatística [Internet]. Cidades e Estados - Florianópolis. Rio de Janeiro: IBGE; 2018. [Acesso 2019 Fev 24]. Disponível em: https://www.ibge.gov.br/cidades-e-estados/sc/florianopolis.html

18. Brasil. Ministério da Saúde. Secretaria de Atenção à Saúde. Departamento de Atenção Básica [Internet]. Nota técnica - Informações sobre as ações e programas do Departamento de Atenção Básica - Florianópolis - SC. Brasília: Ministério da Saúde; 2019 [Acesso 2019 Fev 24]. Disponível em: http://dab2.saude.gov.br/sistemas/notatecnica/frmListaMunic.php

19. von Wangenheim A, Nunes DH. Direct Impact on Costs of the Teledermatology-Centered Patient Triage in the State of Santa Catarina. Florianópolis: Instituto Nacional para Convergência Digital - Universidade Federal de Santa Catarina; 2018. p. 9-13.

20. Spedo SM, Pinto NRS, Tanaka OY. O difícil acesso a serviços de média complexidade do SUS: o caso da cidade de São Paulo, Brasil. Physis. 2010;20(3):953-72. https://doi.org/10.1590/S0103-73312010000300014

21. Stevenson P, Finnane AR, Soyer HP. Teledermatology and clinical photography: safeguarding patient privacy and mitigating medico-legal risk. Med J Aust. 2016;204(5):198-200e1. https://doi.org/10.5694/mja15.00996

22. Silva CS, Souza MB, Duque IA, Medeiros LM, Melo NR, Araújo CA, et al. Teledermatologia: correlação diagnóstica em serviço primário de saúde. An Bras Dermatol. 2009;84(5):489-93. https://doi.org/10.1590/S0365-05962009000500007

23. Tandjung R, Badertscher N, Kleiner N, Wensing M, Rosemann T, Braun RP, et al. Feasibility and diagnostic accuracy of teledermatology in Swiss primary care: process analysis of a randomized controlled trial. J Eval Clin Pract. 2015;21(2):326-31. https://doi.org/10.1111/ jep. 12323

24. Yarak S, Ruiz EES, Pisa IT. A Teledermatologia na Prática Médica. Rev Bras Educ Med. 2017;41(2):346-55. https://doi.org/10.1590/1981$52712015 \mathrm{v} 41 \mathrm{n} 2 \mathrm{rb} 20160042$

25. Fogel AL, Sarin KY. A survey of direct-to-consumer teledermatology services available to US patients: explosive growth, opportunities and controversy. J Telemed Telecare. 2017;23(1):19-25. https://doi.org/10.1177/1357633X15624044 